BMJ Open Sport \& Exercise Medicine

\section{Prevalence of mental health symptoms among male and female Australian professional footballers}

To cite: Kilic 0̈, Carmody S, Upmeijer J, et al. Prevalence of mental health symptoms among male and female Australian professional footballers. BMJ Open Sport \& Exercise Medicine 2021;7:e001043. doi:10.1136/ bmjsem-2021-001043

Accepted 17 July 2021 Check for updates

(c) Author(s) (or their employer(s)) 2021. Re-use permitted under CC BY-NC. No commercial re-use. See rights and permissions. Published by BMJ.

${ }^{1}$ Amsterdam UMC, University of Amsterdam, Department of Orthopaedic Surgery, Amsterdam Movement Sciences Meibergdreef 9, Amsterdam, The Netherlands

${ }^{2}$ Medical Department, Chelsea Football Club, London, The Netherlands

${ }^{3}$ Amsterdam Collaboration on Health \& Safety in Sports (ACHSS), Amsterdam UMC IOC Research Center of Excellence, Amsterdam, The Netherlands ${ }^{4}$ Academic Center for EvidenceBased Sports Medicine (ACES), Amsterdam University Medical Centers, Amsterdam, The Netherlands

${ }^{5}$ Orygen, Parkville, Melbourne, Victoria, Australia

${ }^{6}$ Centre for Youth Mental Health, University of Melbourne, Melbourne, Victoria, Australia ${ }^{7}$ Section Sports Medicine, University of Pretoria, Pretoria, South Africa

${ }^{8}$ Football Players Worldwide (FIFPRO), Hoofddorp, The Netherlands

Correspondence to Dr Vincent Gouttebarge; v.gouttebarge@amsterdamumc. $\mathrm{nl}$

\section{ABSTRACT}

Objective Determining the prevalence of mental health symptoms (MHS) among Australian professional footballers compared with former players. A secondary aim was to assess whether MHS were associated with recent injury and psychological resilience.

Methods This cross-sectional study included 149 male (mean age: 24 years) and 132 female (mean age: 23 years) Australian A-League and W-League professional footballers (study group) and 81 former male footballers (control group, mean age: 39 years) for analysis. MHS and psychological resilience were assessed through validated questionnaires. Severe injuries were assessed through a single question. The adjusted Wald method was used to assess the primary aim. Logistic regression analyses was used to assess the secondary aim.

Results The most prevalent MHS among active footballers and former footballers was sport-related psychological distress (63\%) and alcohol misuse (69\%), respectively. Global psychological distress, sleep disturbance, alcohol misuse and substance misuse were significantly lower among active male footballers than among former players. Increased psychological resilience among active male footballers was associated with a decrease in symptoms of sport-related and global psychological distress, anxiety and depression of $9 \%$, $14 \%, 23 \%$ and $20 \%$, respectively. Increased psychological resilience among female players was associated with $10 \%$ decrease in symptoms of depression. Problem gambling and sleep disturbance was associated with injury in the previous 6 months among active male and female footballers, respectively.

Conclusions MHS are prevalent among active and former professional footballers. Higher level of psychological resilience is associated with decreased reporting of MHS. Severe injury is associated with problem gambling and sleep disturbance.

\section{INTRODUCTION}

Mental health symptoms (MHS) in elite sports have been increasingly examined in the past decade. ${ }^{1}$ Recent meta-analysis showed that the prevalence of MHS ranged from $19 \%$ for alcohol misuse to $34 \%$ for anxiety/depression in active elite athletes, and between $16 \%$ for psychological distress to $26 \%$ for
What are the new findings

Mental health symptoms (MHS) are common among male and female Australian professional footballers as well as among retired male professional footballers.

- Higher level of psychological resilience is associated with decreased reporting of MHS

- Severe injury in the past 6 months is associated with problem gambling and disordered eating.

anxiety/depression in former elite athletes. ${ }^{2}$ Several stressors (such as injuries) contribute to the occurrence of MHS in elite athletes. ${ }^{1}$ Earlier studies have shown that MHS among professional footballers occur subsequently after musculoskeletal injuries. ${ }^{3} 4$ Severely injured European professional footballers were up to seven times more likely to subsequently report MHS than uninjured players. ${ }^{4}$ However, factors such as resilience (ie, positive coping strategies) can protect athletes from developing MHS. ${ }^{1}$

In Australia, Gulliver et al explored the prevalence of MHS among elite athletes from various sports and found that around 45\% reported experiencing symptoms of at least one of the MHS under study. ${ }^{5}$ More recently, Purcell et al found that in a nationally representative sample of currently competing Australian elite athletes from various sports, the reported rates of 'high to very high' psychological distress were elevated in comparison to demographically appropriate community control samples ( $18 \%$ vs $10 \%$, respectively). The extent to which MHSs are prevalent in Australian professional football, as opposed to elite (eg, non-professional, Olympic level) sport, remains unknown, but warrants attention. Even more, it is important to capture the difference in prevalence of MHS between active and former players so that stakeholders can establish whether and when a particular support measure can be offered. 
The primary aim of our study was to determine the prevalence of MHS among male and female Australian professional footballers, drawing comparisons to a group of former players. A secondary aim was to assess whether MHS reported among male and female Australian professional footballers was associated with recent injury and psychological resilience.

\section{METHODS AND ANALYSIS Study design}

Reported in compliance with the Strengthening the Reporting of Observational Studies in Epidemiology statement, ${ }^{7}$ this study was an observational comparative cross-sectional survey.

\section{Study participants}

The study group consists of two separate groups, namely active male and female professional footballers. Inclusion criteria were: (1) being a professional footballer active in the Australian A-League or W-League; (2) $\geq 18$ years and (3) being a member of the Professional Footballers Australia (PFA). The two comparison groups consisted of former male and female Australian professional footballers. Sample size calculation with regard to our primary aim indicated that 130 participants in each study group were needed (power of $80 \%$, CI of $95 \%$; absolute precision of $7.5 \%$ ) under the assumption of an anticipated prevalence of 25\%, ${ }^{128}$ although all PFA active members in the A-League and W-League were invited to participate.

\section{Dependent variables}

MHS were operationalised by measuring domains of psychological distress, anxiety, depression, sleep disturbance, alcohol misuse, substance misuse, disordered eating and gambling. Psychological distress was assessed with the Athlete Psychological Strain Questionnaire (APSQ) and the Kessler-10 (K-10). The APSQ measures sport-related psychological distress through 10 items (eg, 'It was difficult to be around teammates') scored on a 5-point scale (from 1 'none of the time' to 5 'all of the time').$^{9}{ }^{10}$ A total score ranging from 10 to 50 was calculated, with a sum score of $\geq 17$ indicating the presence of sport-related psychological distress. ${ }^{9}{ }^{10}$ The K-10 measures global psychological distress through 10 items (eg, 'During that 4 weeks, how often did you feel tired out for no good reason?') scored on a 5-point scale (from 1 'none of the time' to 5 'all of the time'). ${ }^{11}{ }^{12}$ A total score ranging from 10 to 50 was calculated, with a sum score of $\geq 21$ indicating the presence of global psychological distress. ${ }^{11} 12$ Anxiety was assessed with the General Anxiety Disorder-7 (GAD-7) that relies on seven items (eg, 'Over the last 2 weeks, how often have you been bothered by feeling nervous, anxious, or on edge?') scored on a 4-point scale (from 0 'not at all' to 3 'nearly every day'). ${ }^{1314} \mathrm{~A}$ total score ranging from 0 to 21 was calculated, with higher sum scores indicating greater anxiety severity (0-4: normal; 5-9: mild; 10-14: moderate and $\geq 15$ : (moderately) severe) and a sum score of $\geq 10$ indicating symptoms consistent with anxiety. ${ }^{13}{ }^{14}$ Depression was assessed with the Patient Health Questionnaire 9 that relies on nine items (eg, 'Over the last 2 weeks, how often have you been bothered by little interest or pleasure in doing things?') scored on a 4-point scale (from 0 'not at all' to 3 'nearly every day'). ${ }^{15} 16$ A total sum score ranging from 0 to 27 was calculated, with higher sum scores indicating greater depression severity $(0-4$ : normal; 5-9: mild; $10-14$ : moderate and $\geq 15$ (moderately) severe) and with a sum score of $\geq 10$ indicating symptoms consistent with depression. ${ }^{15} 16$ Sleep disturbance was assessed with the Athlete Sleep Screening Questionnaire that relies on five items (eg, 'During the recent past, how many hours of actual sleep did you get at night?') scored on 4- and 5-point scales. ${ }^{1718}$ A total score ranging from 1 to 17 was calculated, with higher sum scores indicating greater sleep disturbance severity (5-7: mild; 8-10: moderate and $\geq 11$ severe) and with a sum score of $\geq 8$ indicating the presence of sleep disturbance. ${ }^{19}$ Alcohol misuse was assessed with the Alcohol Use Disorders Identification Test Consumption that relies on three items (eg, 'How often do you have a drink containing alcohol?'). ${ }^{19}$ A total score ranging from 0 to 12 was calculated, with a sum score of $\geq 4$ (male) and $\geq 3$ (female) indicating the presence of alcohol misuse. ${ }^{19}$ Substance misuse was assessed with the Cutting Down, Annoyance by Criticism, Guilty Feeling and Eye-openers Adapted to Include Drugs that relies on four items (eg, 'In the last 3 months, have you felt you should cut down or stop using drugs?') scored as yes or no. ${ }^{20} \mathrm{~A}$ total score ranging from 0 to 4 was calculated, with a sum score of $\geq 2$ indicating the presence of substance misuse. ${ }^{21}$ Disordered eating was assessed with the Brief Eating Disorder in Athletes Questionnaire that relies on nine items (eg, 'Over the last 2 weeks, how often have you been bothered by feeling extremely guilty after overeating?') scored on several scales. ${ }^{22}$ A total score ranging from 0 to 18 was calculated, with a sum score of $\geq 4$ indicating the presence of disordered eating. ${ }^{21}$ Gambling was assessed with the NORC Diagnostic Screen for Gambling Disorders for Control, Lying and Preoccupation that relies on three items (eg, "Have there ever been periods lasting 2 weeks or longer when you spent a lot of time thinking about your gambling experiences or planning out future gambling ventures or bets?') scored as yes or no. ${ }^{23} \mathrm{~A}$ total score ranging from 0 to 3 was calculated, with a sum score of $\geq 1$ indicating the presence of problem gambling. ${ }^{23}$ All instruments used (and related cut-off values) to assess MHS are part of the International Olympic Committee (IOC) Sport Mental Health Assessment Tool 1 (SMHAT-1), have been validated in English across different populations (eg, general population, patients, athletes) and have shown good to excellent psychometric properties. $^{21}$

\section{Independent variables}

The total number of severe injuries experienced in the previous 6 months was examined through a single 
Table 1 Descriptive variables of the male and female Australian professional footballers (active and former)

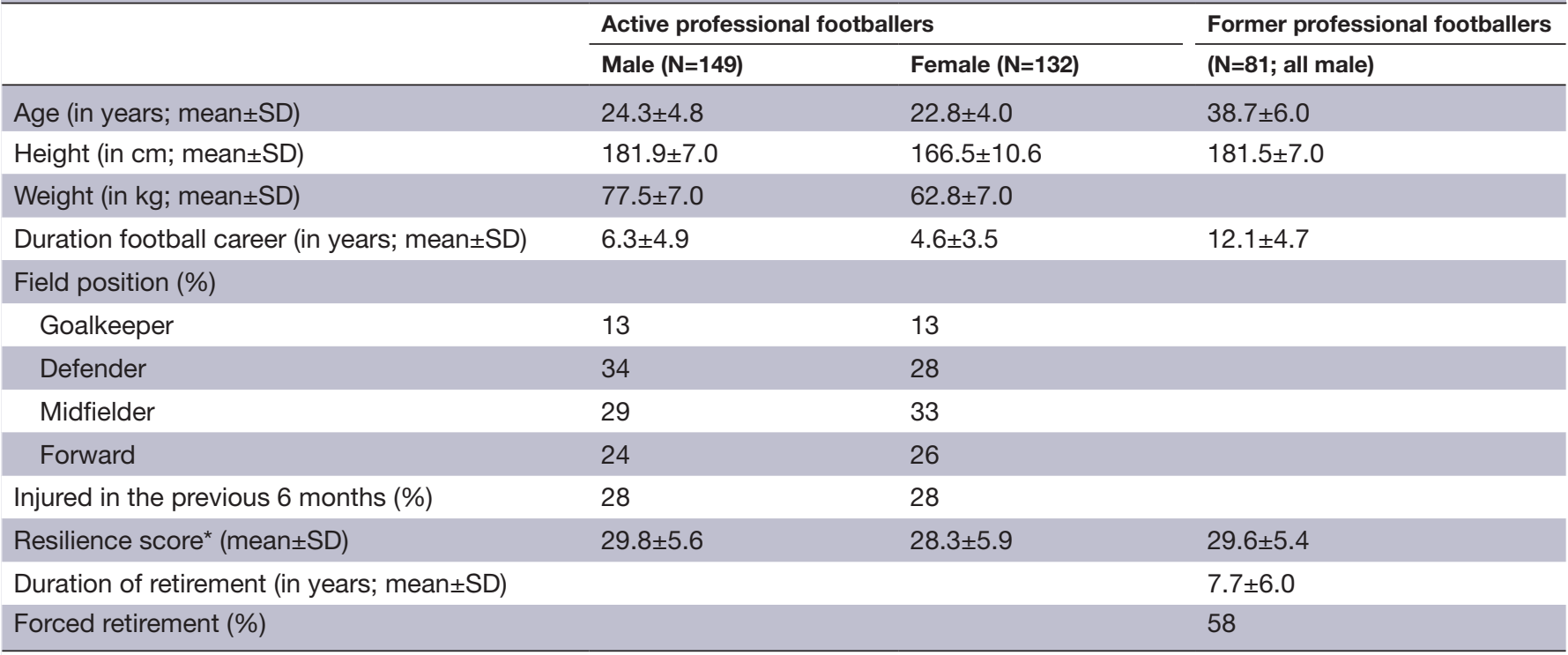

$\mathrm{N}$, number of participants; SD, standard deviation; cm, centimeters; kg, kilograms; \%, percentage.

${ }^{*}$ Ranging from 0 to 40 with higher score indicating greater resilience.

self-report question (eg, 'Did you suffer from a severe musculoskeletal injury (bone, muscle, tendon, ligaments) in the past 6 months?'). We defined severe injury as an injury that involved the musculoskeletal system (bone, joint, ligament, muscles, tendons) that occurred during team activities and led to either training or match absence for more than 28 days (this definition was clearly stated to the participants). ${ }^{24}$ Psychological resilience was assessed with the validated Connor-Davidson Resilience Scale (CD-RISC) that relies on 10 items scored on a 5-point scale. ${ }^{24} \mathrm{~A}$ total score ranging from 0 to 40 was calculated, with a higher score indicating greater psychological resilience. ${ }^{25}$

\section{Procedures}

An electronic anonymous survey in English was compiled (SurveyMonkey), including several descriptive variables (eg, age, gender, height, weight, duration of professional football career, field position). Information about the study was provided to potential participants by the PFA's staff during their club visits (study group) or via email (comparison group). If interested, participants provided their electronic informed consent and were given access to the online survey, which took approximately $20 \mathrm{~min}$ to complete. Once completed, the electronic surveys were automatically saved on a secured server that only the principal investigator could access. Participants did not receive any incentive to participate in the study. Data were collected from December 2019 to February 2020.

\section{Statistical analyses}

The statistical software IBM SPSS V.26.0 for Apple Mac was used for data analysis. Descriptive analyses (mean, $\mathrm{SD}$, frequency and range) were performed for all descriptive variables. For our primary aim, prevalence of MHS (expressed as percentage) was calculated as the proportion of the number of participants with the condition relative to the total number of participants, using the adjusted Wald method for $95 \% \mathrm{CI}$ as the group consisted of less than 150 participants. ${ }^{8}$ Prevalence was also calculated for each level of severity for anxiety, depression and sleep disturbance. Differences between study and comparison groups in prevalence of MHS were determined with $\chi^{2}$ tests.$^{8}$ For our secondary aim, logistic regression analyses (level of statistical significance: $\mathrm{p}<0.05)$ expressed as OR and 95\% CI were performed to explain the potential association of both independent variables, namely injuries (dichotomous; injured or not in the previous 6 months) and psychological resilience (continuous), with MHS, the dependent variable. ${ }^{8}$ This calculation was done separately for both groups (active male and female players).

\section{Patient and public involvement}

Participants were not involved in the design, or conduct, or reporting or dissemination plans of the study. However, participants' representatives were consulted and provided input to the recruitment's strategy.

\section{RESULTS}

\section{Participants}

The PFA contacted 280 male and 170 female active professional footballers. From those, 149 male (mean age of 24 years) and 132 female (mean age of 23 years) players gave their informed consent (overall response rate of $62 \%$ ). Participants played professional football for 5 years on average. In the previous 6 months $28 \%$ reported to have suffered from $\geq 1$ severe injuries. The PFA contacted 400 former professional footballers for the comparison group, 89 (81 male, 8 female) gave their informed consent (response rate of 22\%). Because 
Table 2 Prevalence $(\% ; 95 \% \mathrm{Cl})$ of mental health symptoms in male and female Australian professional footballers (active and former)

\begin{tabular}{|c|c|c|c|}
\hline & Active professional $\mathrm{f}$ & & Former professional footballers \\
\hline & Male $(\mathrm{N}=149)$ & Female $(\mathrm{N}=132)$ & $(\mathrm{N}=81)$ \\
\hline Sport-related psychological distress & $52.0(44.0$ to 60.1$)$ & $62.9(54.6$ to 71.1$)$ & \\
\hline Global psychological distress & 9.5 (5.6 to 15.4$)$ & 18.9 (13.1 to 26.5$)$ & $26.3(17.1 \text { to } 35.0)^{*}$ \\
\hline Anxiety & 4.7 (2.1 to 9.6$)$ & 8.3 (4.6 to 14.5$)$ & 11.3 (5.3 to 18.5$)$ \\
\hline Mild & 22.3 (15.9 to 29.9 ) & 32.6 (25.2 to 41.0$)$ & 38.8 (30.2 to 50.2$)$ \\
\hline Moderate & 2.7 (0.8 to 7.0$)$ & 6.0 (2.9 to 11.7$)$ & 9.0 (3.7 to 15.8$)$ \\
\hline Severe & $2.0(0.4$ to 5.6$)$ & $2.3(0.5$ to 6.8$)$ & $2.3(0.1$ to 8.4$)$ \\
\hline Depression & 6.8 (3.6 to 12.1$)$ & $10.6(6.3$ to 17.1$)$ & 12.5 (7.0 to 21.2$)$ \\
\hline Mild & 20.3 (14.5 to 27.7$)$ & 35.6 (27.9 to 44.1$)$ & 26.1 (18.0 to 36.2$)$ \\
\hline Moderate & $5.4(2.4$ to 10.4$)$ & 8.3 (4.6 to 14.5$)$ & 9.1 (4.5 to 17.2$)$ \\
\hline Severe & $1.4(0.1$ to 4.8$)$ & 2.3 (0.5 to 6.8$)$ & $3.4(0.8$ to 10.0$)$ \\
\hline Sleep disturbance & $12.2(7.8$ to 18.5$)$ & 32.6 (25.2 to 41.0$)$ & $32.5(23.0 \text { to } 42.2)^{*}$ \\
\hline Mild & $87.8(81.1$ to 92.6$)$ & 67.4 (59.0 to 74.8$)$ & 67.5 (57.8 to 77.0$)$ \\
\hline Moderate & 6.8 (3.6 to 12.1$)$ & 23.5 (17.0 to 31.4$)$ & 21.1 (13.3 to 30.1$)$ \\
\hline Severe & 5.4 (2.6 to 10.4$)$ & 9.1 (5.2 to 15.4$)$ & $11.4(6.1$ to 19.9$)$ \\
\hline Alcohol misuse & 50.7 (42.7 to 58.6$)$ & 43.8 (35.6 to 52.4 ) & $68.8(60.2 \text { to } 79.0)^{*}$ \\
\hline Substance misuse & 2.0 (0.4 to 6.1$)$ & 1.5 (0.1 to 5.8$)$ & $10.0(6.1 \text { to } 19.9)^{\star}$ \\
\hline Problem gambling & 23.6 (17.5 to 31.1$)$ & 2.3 (0.5 to 6.9$)$ & 32.5 (23.0 to 42.2 ) \\
\hline Disordered eating & 35.1 (27.9 to 43.1 ) & 43.8 (35.6 to 52.4 ) & 40.0 (28.1 to 48.0$)$ \\
\hline
\end{tabular}

$\mathrm{N}$, number of participants; \%, percentage; $\mathrm{Cl}$, confidence of interval.

*Significant difference $(p<0.01)$ with active male players.

of this limited number of female former players, only the male former professional footballers (mean age of 39 years) were used for further analyses. These former male players had played professional football for 12 years on average and were retired for 8 years $(58 \%$ forced to retire). Participants' demographic and other characteristics are presented in table 1 .

\section{Prevalence}

All prevalence rates of MHS are presented in table 2. In both male and female professional footballers, sportpsychological distress was common, prevalence reaching $52 \%$ and $63 \%$, respectively. Prevalence of other MHS ranged from $2 \%$ for substance misuse to $51 \%$ for alcohol misuse among male players, and from $2 \%$ for substance misuse to $44 \%$ for alcohol misuse and disordered eating among female players. In the comparison group, prevalence of MHS ranged from $10 \%$ for substance misuse to $69 \%$ for alcohol misuse. The prevalence of global psychological distress $\left(10\right.$ vs $\left.26 \% ; \chi^{2}=11.267, \mathrm{df}=1 ; \mathrm{p}<0.01\right)$, sleep disturbance ( 12 vs $\left.33 \% ; \chi^{2}=13.792, \mathrm{df}=1 ; \mathrm{p}<0.01\right)$, alcohol misuse ( 51 vs $\left.69 \% ; \chi^{2}=6.922, \mathrm{df}=1 ; \mathrm{p}<0.01\right)$ and substance misuse ( 2 vs $10 \% ; \chi^{2}=7.189$, $\mathrm{df}=1 ; \mathrm{p}<0.01$ ) was significantly lower in the group of active male players than in the comparison group.

\section{Associations}

Injury in the previous 6 months was associated with problem gambling (OR 2.28; 95\% CI 1.03 to 5.02) but not with other MHS among male professional footballers. Male players with a higher psychological resilience were less likely to report sport-related psychological distress (OR 0.91; 95\% CI 0.85 to 0.97 ), global psychological distress (OR $0.86 ; 95 \%$ CI 0.77 to 0.96 ), anxiety (OR $0.77 ; 95 \%$ CI 0.65 to 0.90 ) and depression (OR 0.80 ; $95 \%$ CI 0.69 to 0.91 ). Meaning that for a unit increase in psychological resilience, the odds of reporting sportrelated and global psychological distress, anxiety and depression decreased by $9 \%, 14 \%, 23 \%$ and $20 \%$, respectively. Among female professional footballers, injury in the previous 6 months was associated with sleep disturbance (OR 2.65; 95\% CI 1.20 to 5.85), while an unit increase in psychological resilience was associated with a $10 \%$ decrease in symptoms of depression (OR 0.90; $95 \%$ CI 0.82 to 0.98 ). No other associations were found in female players.

\section{DISCUSSION}

The prevalence of MHS among the participants within this study was up to $69 \%$. The most prevalent MHS among active male and female professional footballers was sport-related psychological distress with, respectively, $52 \%$ and $63 \%$. The most common MHS among former footballers was $69 \%$ for alcohol misuse. Overall, the prevalence of MHS such as global psychological distress, sleep disturbance, alcohol misuse and other substance misuse were significantly lower in the group of active 
male players vs former professional footballers. Injury in the 6 months prior to the survey was a salient risk factors for both genders, but was associated with a higher odds of gambling in males, and greater sleep disturbance in females. Higher psychological resilience was associated with lower sports-related and global psychological distress, anxiety and depression in males and a decrease in symptoms of depression in females. There was no significant association in both groups for other symptoms of MHS.

\section{Comparison with other studies}

Compared with prior meta-analytic research by Gouttebarge et al (that included various sport types (eg, cricket, football, handball, ice hockey, rugby, boxing, judo, gymnastics, rowing, swmiming), the results of our study show somewhat higher prevalence of MHS. ${ }^{2}$ The prevalence of MHS among current and former elite athletes (males and females) in the review by Gouttebarge et al ranged from $19 \%$ for alcohol misuse to $34 \%$ for anxiety/ depression among current players and $16 \%$ for distress to $26 \%$ for anxiety/depression among former athletes. ${ }^{2}$ In the present study, MHS reached up to $63 \%$ for sports related psychological distress among active players as for retired players the prevalence of MHS reached op to $69 \%$ for alcohol misuse. Cautiousness is warranted as the population characteristics and representativeness in both studies may differ, as in this study only football players are involved. Also, the association between injuries and MHS, as shown in previous studies (eg, distress, anxiety, sleeps disturbance) was not in accordance with the results of this study as injury in the previous 6 months was only associated with gambling. ${ }^{24}$ Junge $e t$ al showed a $13 \%$ and $7 \%$ prevalence of depressive symptoms in first Swiss league female footballers (average age of 21) and male footballers (average age of 25), respectively. ${ }^{26}$ The presence of depressive symptoms in our study was $11 \%$ and $7 \%$ among female and male footballers respectively, showing somewhat similar results. Among female players from the German First League, Prinz et al found a higher prevalence of depression symptoms, namely $32 \% .{ }^{27}$ This could be explained potentially by the use of different instruments to assess MHSs or different characteristics of each sample.

Compared with other elite-level sports Drew et alshowed a substantial prevalence of sleep disturbances (49\% poor sleep, 22\% sleepiness) and poor mental health among Olympic athletes, male and female combined (average age 26 and 24 respectively). They also stated that good mental health, resilience and sleep quality appear to be important factors involved in remaining healthy. ${ }^{28}$ Even though the present study involves a different population of only football players, it does shows also a substantial prevalence of MHS, wherein sleep disturbance presents a prevalence range $(95 \% \mathrm{CI})$ of $12.2 \%(7.8 \%$ to $18.5 \%)$ to $35.6 \%(25.2 \%$ to $41.0 \%)$ among active male and female footballers, respectively.
Slade et al assessed the mental health well-being of the Australian general populations through a self-report survey (age 18 years and older).$^{29}$ Even though Slade $e t$ al used different screening tools to assess mental health status among the Australian general population, they also used the K10 scale to assess distress corresponding with the present study. ${ }^{29}$ The prevalence of GADs was $2 \%$ and $4 \%$ for both male and females. ${ }^{29}$ The prevalence of depression was $3 \%$ and $5 \%$ for male and females, respectively. ${ }^{29}$ Drug use disorder was prevalent among $1 \%$ of males and $0.5 \%$ of females while alcohol use disorder was, respectively, $4 \%$ and $2 \% .{ }^{29}$ Any substance use disorder was $7 \%$ and $3 \%$ for male and females respectively. ${ }^{29}$ The active and former athlete population in the present study shows higher prevalence rates of symptoms of anxiety, depression, alcohol-use disorders and drug-use disorder.

In our study, we found that previous injury was a contributing factor to problem gambling among active male footballers and to disordered eating among active female footballers. Our findings are in line with the scientific literature showing that injury and MHS might intersect. ${ }^{1}$ Gouttebarge et al concluded that the number of severe musculoskeletal injuries and surgeries during a career was positively correlated and associated with MHS among male European professional footballers. ${ }^{3}$ Even more, Kiliç et al showed that previous severe injuries were associated with the onset of MHS during the subsequent season (relative risks up to 7 ). ${ }^{4}$ This confirms that injury leads to cognitive, emotional and behavioural responses among elite athletes, including disordered gambling, changes in appetite and disordered eating. ${ }^{1}$

\section{Limitations}

Measurement of MHS through self-report might be a potential limitation as it measures only symptoms being consistent with mental health disorders and as it can lead to recall bias. Also for the collection of injury data, the method of self-report is not in accordance with the Fuller et al consensus statement. ${ }^{24}$ In our study, non-response analysis could not be conducted, because potential participants were invited for the study by the PFA with the procedure being blinded to the research team for privacy and confidentiality reasons. This might have led to selection bias and thus have influenced the external validity of our results. Another potential source of bias might be that football players with a particular interest in MHS were more prone to participate. The present study was done through anonymous recruitment and validated scales in order to improve the objectivity of the participants towards their own mental health, as MHS are a rather taboo subject in professional sports such as football. ${ }^{30}$ However a possible bias may arise from this ongoing taboo surrounding MHS among elite athletes, which could lead to a possible underestimation in the extent of MHS. An additional limitation is that there was no reference or comparison group from the general population included in the present study. In addition to a group of former players, a reference or comparison 
group from a non-athlete population, matched for age and gender, might have provided the possibility to appreciate potential differences and make other interpretations. Furthermore the number of participants within the former players group did not meet the power criteria ( $\mathrm{N}=130$ per group). The low number of former female participants in particular precluded further analyses with this group. A specific explanation for the low number of former female players could be that the female football industry is only just starting to develop and to be unionised, making them more difficult to track and approach for participation. Also the cross-sectional set up of this study does not allow to assess any causal relationship between the variables. Nonetheless, the results of this study provides a foundation for future research.

\section{Recommendations}

In several past studies different tools and populations were used to assess the symptoms of MHS, creating heterogeneity and complexity in the ability to easily compare between studies. ${ }^{2}$ A recommendation for further studies is to use the IOC Consensus Statement on mental health in elite athletes as a more evidence based and standardised approach to this subject is warranted. ${ }^{130}$ Future studies should have a longitudinal design of several years making it possible to assess causalities. Furthermore, there should be more attention to enrolling female participants in studies about MHS among active and retired athletes.

The prevalence of MHS among footballers have shown to be common, meaning that support and a multidisciplinary approach by mental health professionals as well as sport medicine physicians should be standard care. The IOC SMHAT-1 and The IOC Sport Mental Health Recognition Tool 1 (SMHRT-1) should be used to identify and help athletes that are at risk for developing MHS as well as increasing their psychological resilience. ${ }^{21}$ The SMHAT-1 identifies athletes that are in need for mental health treatment. This tool should be used by the sports medicine physician and/or licensed and registered mental health professionals in order to set up a multidisciplinary approach in tackling the MHS of the footballers. ${ }^{21}$ Psychological resilience is a significant protective factor against MHS according to this study. Family, teammates, coaches and friends known as the footballer players' entourage have been identified as essential support in the mental well-being. With the SMHRT-1 the footballers' entourage can facilitate in the early detection of MHSs and support early help-seeking by footballers from their sports medicine physician or psychologist. ${ }^{21}$ Education about the negative effects of alcohol misuse should be given to football players. ${ }^{31} 32$ This might help improving coping behaviour as it is also shown that sleeping problems or anxiety may lead to alcohol misuse and vice versa. ${ }^{31}$ Early detection and if needed treatment of MHS can serve to improve the athletes psychological resilience, performance and quality of life.

In professional football, an after career consultation has recently been introduced. ${ }^{33}$ In an earlier pilot study, such an intervention was positively evaluated by former players. ${ }^{2}{ }^{34}$ In particular, athletes forced into early retirement, but also voluntarily retired athletes, could benefit from programmes preparing them for post-elite sport times (eg, seminars, mental and life skills training about career transition). ${ }^{233} \mathrm{~A}$ smooth transition into retirement could prevent the development of MHS among retired athletes and improve their psychological resilience.

\section{CONCLUSION}

The high prevalence rates of MHS among active and former professional footballers indicate that attention to this matter is warranted. The present study showed that increase in psychological resilience was protective against MHS. Several tools are available to recognise symptoms of MHS in an early stage, making a multidisciplinary approach possible to treat and educate athletes in order to improve their well-being, performance, quality of life as well as their psychological resilience and coping behaviour. For retired athletes, an after career consultation could help enable a smooth transition into retirement.

\section{Twitter Özgür Kilic @KilicOzgur_and Vincent Gouttebarge @VGouttebarge}

Acknowledgements The authors would like to thank the staff of Professional Footballers Australia (PFA) for their support in the study. We are grateful to all active and former professional footballers who participated in the study.

Contributors VG and GMMJK were responsible for the study design and data collection. All authors were involved in the data analysis and data interpretation. ÖK drafted the initial version of the manuscript, with critical review provided by all authors. All authors approved the final version of the manuscript.

Funding The authors have not declared a specific grant for this research from any funding agency in the public, commercial or not-for-profit sectors.

Competing interests None declared.

Patient and public involvement Patients and/or the public were not involved in the design, or conduct, or reporting, or dissemination plans of this research.

Patient consent for publication Not required.

Ethics approval Ethical approval was provided by the Medical Ethics Review Committee of the Amsterdam University Medical Centre (W18_340\#18.387; Amsterdam, The Netherlands), and the study was conducted in accordance with the Declaration of Helsinki (2013).

Provenance and peer review Not commissioned; externally peer reviewed. Data availability statement Data are not available.

Open access This is an open access article distributed in accordance with the Creative Commons Attribution Non Commercial (CC BY-NC 4.0) license, which permits others to distribute, remix, adapt, build upon this work non-commercially, and license their derivative works on different terms, provided the original work is properly cited, appropriate credit is given, any changes made indicated, and the use is non-commercial. See: http://creativecommons.org/licenses/by-nc/4.0/.

\section{ORCID iDs}

Özgür Kilic http://orcid.org/0000-0002-8106-6380

Sean Carmody http://orcid.org/0000-0001-8683-5532

Simon Rice http://orcid.org/0000-0003-4045-8553

Vincent Gouttebarge http://orcid.org/0000-0002-0126-4177

\section{REFERENCES}

1 Reardon CL, Hainline B, Aron CM, et al. Mental health in elite athletes: international Olympic Committee consensus statement (2019). Br J Sports Med 2019;53:667-99.

2 Gouttebarge V, Castaldelli-Maia JM, Gorczynski P, et al. Occurrence of mental health symptoms and disorders in current and former elite 
athletes: a systematic review and meta-analysis. Br J Sports Med 2019;53:700-6.

3 Gouttebarge V, Aoki H, Ekstrand J, et al. Are severe musculoskeletal injuries associated with symptoms of common mental disorders among male European professional footballers? Knee Surg Sports Traumatol Arthrosc 2016;24:3934-42.

4 Kiliç Ö, Aoki H, Goedhart E, et al. Severe musculoskeletal time-loss injuries and symptoms of common mental disorders in professional soccer: a longitudinal analysis of 12-month follow-up data. Knee Surg Sports Traumatol Arthrosc 2018;26:946-54.

5 Gulliver A, Griffiths KM, Mackinnon A, et al. The mental health of Australian elite athletes. J Sci Med Sport 2015;18:255-61.

6 Purcell R, Rice S, Butterworth M, et al. Rates and correlates of mental health symptoms in currently competing elite athletes from the Australian National high-performance sports system. Sports Med 2020;50:1683-94

7 Vandenbroucke JP, von Elm E, Altman DG, et al. Strengthening the reporting of observational studies in epidemiology (STROBE) explanation and elaboration. Epidemiology 2007;18:805-35.

8 Woodward M. Epidemiology : study design and data analysis. Boca Raton: CRC Press, 2014.

9 Rice S, Olive L, Gouttebarge V, et al. Mental health screening: severity and cut-off point sensitivity of the athlete psychological strain questionnaire in male and female elite athletes. BMJ Open Sport Exerc Med 2020;6:e000712.

10 Rice SM, Parker AG, Mawren D. Preliminary psychometric validation of a brief screening tool for athlete mental health among male elite athletes: the athlete psychological strain questionnaire. Int $J$ Sport Exercise Psy 2019:1-16.

11 Kessler RC, Andrews G, Colpe LJ, et al. Short screening scales to monitor population prevalences and trends in non-specific psychological distress. Psychol Med 2002;32:959-76.

12 Prinsen CAC, Mokkink LB, Bouter LM, et al. COSMIN guideline for systematic reviews of patient-reported outcome measures. Qual Life Res 2018;27:1147-57.

13 Plummer F, Manea L, Trepel D, et al. Screening for anxiety disorders with the GAD-7 and GAD-2: a systematic review and diagnostic metaanalysis. Gen Hosp Psychiatry 2016;39:24-31.

14 Spitzer RL, Kroenke K, Williams JBW, et al. A brief measure for assessing generalized anxiety disorder: the GAD-7. Arch Intern Med 2006;166:1092-7.

15 Kroenke K, Spitzer RL, Williams JB. The PHQ-9: validity of a brief depression severity measure. J Gen Intern Med 2001;16:606-13.

16 Moriarty AS, Gilbody S, McMillan D, et al. Screening and case finding for major depressive disorder using the patient health questionnaire (PHQ-9): a meta-analysis. Gen Hosp Psychiatry 2015;37:567-76.

17 Driller MW, Mah CD, Halson SL. Development of the athlete sleep behavior questionnaire: a tool for identifying maladaptive sleep practices in elite athletes. Sleep Sci 2018;11:37-44.

18 Samuels C, James L, Lawson D, et al. The athlete sleep screening questionnaire: a new tool for assessing and managing sleep in elite athletes. Br J Sports Med 2016;50:418-22.

19 Dawson DA, Grant BF, Stinson FS, et al. Effectiveness of the derived alcohol use disorders identification test (AUDIT-C) in screening for alcohol use disorders and risk drinking in the US general population. Alcohol Clin Exp Res 2005;29:844-54.

20 Brown RL, Rounds LA. Conjoint screening questionnaires for alcoho and other drug abuse: criterion validity in a primary care practice. Wis Med $J$ 1995;94:135-40.

21 Gouttebarge V, Bindra A, Blauwet C, et al. International Olympic Committee (IOC) sport mental health assessment tool 1 (SMHAT-1) and sport mental health recognition tool 1 (SMHRT-1): towards better support of athletes' mental health. $\mathrm{Br} J$ Sports Med 2021;55:30-7.

22 Martinsen M, Holme I, Pensgaard AM, et al. The development of the brief eating disorder in athletes questionnaire. Med Sci Sports Exerc 2014;46:1666-75.

23 Toce-Gerstein M, Gerstein DR, Volberg RA. The NODS-CLiP: a rapid screen for adult pathological and problem gambling. J Gamb/ Stud 2009;25:541-55.

24 Fuller CW, Ekstrand J, Junge A, et al. Consensus statement on injury definitions and data collection procedures in studies of football (soccer) injuries. Br J Sports Med 2006;40:193-201.

25 Connor KM, Davidson JRT. Development of a new resilience scale: the Connor-Davidson resilience scale (CD-RISC). Depress Anxiety 2003;18:76-82

26 Junge A, Feddermann-Demont N. Prevalence of depression and anxiety in top-level male and female football players. BMJ Open Sport Exerc Med 2016;2:e000087.

27 Prinz B, Dvořák J, Junge A. Symptoms and risk factors of depression during and after the football career of elite female players. BMJ Open Sport Exerc Med 2016;2:e000124.

28 Drew M, Vlahovich N, Hughes D, et al. Prevalence of illness, poor mental health and sleep quality and low energy availability prior to the 2016 summer Olympic Games. Br J Sports Med 2018;52:47-53.

29 Slade T, Johnston A, Oakley Browne MA, et al. 2007 national survey of mental health and wellbeing: methods and key findings. Aust N Z J Psychiatry 2009;43:594-605.

30 Hainline B, Reardon CL. Breaking a taboo: why the International Olympic Committee convened experts to develop a consensus statement on mental health in elite athletes. $\mathrm{Br} J$ Sports Med 2019;53:665-6.

31 Park S-Y, Oh M-K, Lee B-S, et al. The effects of alcohol on quality of sleep. Korean J Fam Med 2015;36:294-9.

32 Anker JJ, Kushner MG. Co-occurring alcohol use disorder and anxiety: bridging psychiatric, psychological, and neurobiological perspectives. Alcohol Res 2019;40. doi:10.35946/arcr.v40.1.03. [Epub ahead of print: 30 Dec 2019].

33 Carmody S, Jones C, Malhotra A, et al. Put out to pasture: what is our duty of care to the retiring professional footballer? Promoting the concept of the 'exit health examination' (EHE). Br J Sports Med 2019;53:788-9.

34 Gouttebarge V, Goedhart E, Kerkhoffs G. Empowering the health of retired professional footballers: the systematic development of an after career consultation and its feasibility. BMJ Open Sport Exerc Med 2018;4:e000466. 